\title{
AUDIT
}

\section{Management of spontaneous pneumothorax-a Welsh survey}

\author{
J H Yeoh, S Ansari, I A Campbell
}

\begin{abstract}
The authors sought to determine to what degree current practice by hospital physicians and accident and emergency (A\&E) departments in Wales conformed to the British Thoracic Society's guidelines for the management of spontaneous pneumothorax. Questionnaires were posted to all consultants involved in emergency medical admissions in Wales (149 consultant physicians and $23 \mathrm{~A} \& \mathrm{E}$ consultants) of whom 101 (59\%) replied. Only 45\% used the classification, "small, moderate, or complete" to describe the size of pneumothorax. Just $44 \%$ would do as recommended by the British Thoracic Society and discharge an asymptomatic patient with a primary pneumothorax and $34 \%$ would discharge a patient with a primary pneumothorax after successful aspiration. Only $20 \%$ were prepared to try aspiration initially for a secondary pneumothorax with a complete lung collapse. Thirty four per cent would follow the recommendation to remove a chest drain without prior clamping of the tube 24 hours after bubbling had stopped. In the event of a persistent air leak $69 \%$ would refer patients or seek a specialist opinion. Physicians with an interest in respiratory medicine tolerated persistent air leaks for significantly longer than did nonrespiratory physicians (median of $7 v 5$ days, $p=0.001$ ). The survey indicates that fewer than expected consultant physicians and $A \& E$ consultants in Wales manage spontaneous pneumothoraces in the way recommended by the guidelines. Physicians with an interest in respiratory medicine tended to comply with these guidelines more than general physicians with interests other than respiratory medicine or $A \& E$ consultants but the trend was not significant at the $5 \%$ level. It is felt that the guidelines should be disseminated more widely, ensuring that emergency admissions units and $A \& E$ departments have copies on display or easily accessible, and that they could be expanded to cover other aspects such as timing for surgery. (Postgrad Med F 2000;76:496-500)
\end{abstract}

Keywords: pneumothorax; non-compliance with guidelines
Spontaneous pneumothorax is a common problem, with an incidence of 8/100 000 persons per year. ${ }^{1}$ The objective of treatment, if required, is to re-expand the lung, usually by aspiration with a fine bore cannula or placement of an intercostal drain. Initial assessment and treatment usually takes place in accident and emergency (A\&E) departments or in the medical admissions units or general medical wards. For patients with persistent air leak (PAL), bilateral or recurrent pneumothoraces thoracic surgery may be required. The British Thoracic Society (BTS) produced guidelines for the management of spontaneous pneumothorax in 1993. ${ }^{2}$ We set out to determine the current practice of hospital physicians and $\mathrm{A} \& \mathrm{E}$ departments in Wales by means of a postal questionnaire and to compare the results with the BTS guidelines.

\section{Methods}

In the spring of 1998 we conducted a postal survey of all consultant physicians and A\&E consultants involved in medical emergency admissions in Wales, sending questionnaires to 149 consultant physicians and 23 A\&E consultants. They were asked to answer 13 questions concerning the management of spontaneous pneumothoraces. A covering letter asked for the questionnaire to be returned at the same time but separately from a chit which indicated agreement to participate so as to enable identification of non-responders and still allow anonymous participation. One reminder was sent to those who had not responded after three weeks. A copy of the questionnaire is available from the authors. The data were analysed with $\chi^{2}$ and Mann-Whitney tests, using SPSS 8.0 for Windows.

\section{Results}

We received 101 replies, a 59\% response rate (table 1). There was no apparent difference in the response rates between

Table 1 Number of consultants surveyed and the number of responders

\begin{tabular}{lcl}
\hline Specialty & No surveyed & \% Responded \\
\hline Respiratory physicians & 29 & 66 \\
Non-respiratory physicians & 120 & 54 \\
A\&E consultants & 23 & 44 \\
Not specified & - & 07 \\
Total & 172 & 59 \\
\hline
\end{tabular}


Table 2 Initial management of spontaneous pneumothorax: compliance with the BTS guidelines

\begin{tabular}{llll}
\hline & $\begin{array}{l}\text { Male, 25 years old, small } \\
\text { primary pneumothorax, not } \\
\text { breathless - discharged with } \\
\text { outpateint follow up (\%) }\end{array}$ & $\begin{array}{l}\text { Male, 40 years old, moderate } \\
\text { primary pneumothorax, } \\
\text { breathless treated with } \\
\text { initial simple aspiration (\%) }\end{array}$ & $\begin{array}{l}\text { Male, 55 years old, large } \\
\text { secondary pneumothorax, } \\
\text { breathless - treated with } \\
\text { initial simple aspiration (\%) }\end{array}$ \\
Specialty & 58 & 84 & 16 \\
\hline Respiratory physicians $(\mathrm{n}=19)$ & 37 & 68 & 19 \\
Non-respiratory physicians ( $=65)$ & 50 & 80 & 50 \\
A\&E consultants $(\mathrm{n}=10)$ & 57 & 71 & 0 \\
Not specified $(\mathrm{n}=7)$ & 56 & 72 & 20 \\
Total $(\mathrm{n}=101)$ & 0.235 & 0.331 & 0.062 \\
$\mathrm{p}$ Value (Pearson $\left.\chi^{2}\right)$ & & & \\
\hline
\end{tabular}

hospitals with urban and rural catchment areas, or from the teaching hospitals in Cardiff. Percentages, henceforth, relate to the respondents only.

ESTIMATION OF SIZE OF PNEUMOTHORAX BY CHEST RADIOGRAPH

Only $45 \%$ graded the size of the pneumothorax as "small, moderate, or complete" (respiratory physicians $47 \%$, non-respiratory physicians $45 \%$, and $\mathrm{A} \& \mathrm{E}$ consultants $50 \%$, $\mathrm{p}=0.939$ ), whereas $45 \%$ graded the pneumothorax in terms of a percentage, $5 \%$ estimated the size in "thirds", 3\% used diagrams, and $2 \%$ gave more than one answer or did not answer.

INITIAL MANAGEMENT OF PNEUMOTHORACES With a small, primary spontaneous pneumothorax (that is, no other underlying lung disease) in a 25 year old patient who was not breathless, only $44 \%$ would conform with the BTS suggestion to discharge the patient with arrangements for outpatient follow up (respiratory physicians $58 \%$, non-respiratory physicians $37 \%$, and A\&E consultants $50 \%$, $\mathrm{p}=0.235)$. For a moderate, primary spontaneous pneumothorax in a 40 year old smoker who was significantly breathless, $72 \%$ would initially aspirate the pneumothorax (respiratory physicians $84 \%$, non-respiratory physicians $68 \%$, and $\mathrm{A} \& \mathrm{E}$ consultants $80 \%$, $\mathrm{p}=0.331$ ). Against the guidelines, $24 \%$ would use an intercostal drain initially. For a complete secondary spontaneous pneumothorax (that is, associated with a pre-existing underlying lung disease) in a 55 year old patient only one in five would try aspiration initially (respiratory physicians 16\%, nonrespiratory physicians $18 \%$, and A\&E consultants $50 \%, p=0.062$ ), whereas the guidelines recommend percutaneous aspiration as the initial treatment of choice in this situation (table 2).

The BTS guidelines advise that patients with a primary spontaneous pneumothorax in whom simple aspiration has been successful

Table 3 Management plans after successful aspiration: compliance with the BTS guidelines

\begin{tabular}{llc}
\hline & $\begin{array}{l}\text { Primary pneumothorax-was } \\
\text { discharged with outpatient } \\
\text { follow up (\%) }\end{array}$ & $\begin{array}{l}\text { Secondary pneumothorax-was } \\
\text { admitted for overnight inpatient } \\
\text { observation (\%) }\end{array}$ \\
\hline Respiratory physician & 47.4 & 89.5 \\
Non-respiratory physician & 29.2 & 96.9 \\
A\&E consultant & 20.0 & 90.0 \\
Not specified & 57.1 & 100.0 \\
Total & 33.7 & 95.0 \\
\hline
\end{tabular}

can be discharged and followed up in the outpatient clinic but two thirds of our consultants would admit the patient for observation overnight, only $34 \%$ indicating that they would discharge the patient with a follow up in the outpatient department (respiratory physicians $47 \%$, non-respiratory physicians $29 \%$, and A\&E consultants $20 \%, p=0.228$ ). However, the guidelines are followed better for patients with secondary spontaneous pneumothorax: after a successful aspiration $95 \%$ would admit the patient for at least overnight observation (respiratory physicians $89 \%$, non-respiratory physicians $97 \%$, and A\&E consultants $90 \%$, $\mathrm{p}=0.0349$; table 3).

\section{INTERCOSTAL DRAINS}

Since A\&E consultants are not normally involved with further care of the patient, they are not included in the results concerning continued care of the patient or intercostal drain.

If the insertion of an intercostal drain results in cessation of air leak and full expansion of the lung, only $34 \%$ would act in accordance with the guidelines and without first clamping the tube, remove the intercostal drain 24 hours after it stops bubbling (respiratory physicians $53 \%$, non-respiratory physicians $29 \%$, $\mathrm{p}=0.059)$. Fifty eight per cent would clamp the intercostal drain before subsequent removal (respiratory physicians $42 \%$, non-respiratory physicians $63 \%, p=0.103)$. Thirty seven per cent would not wait for the intercostal drain to stop bubbling for 24 hours before removing or clamping it (respiratory physicians 26\%, non-respiratory physicians $40 \%, \mathrm{p}=0.277$; table 4).

Forty per cent thought that suction should only be applied if there was a PAL (respiratory physicians $42 \%$, non-respiratory physicians $40 \%, p=0.869)$. Six per cent thought that suction should never be applied and $8 \%$ were uncertain regarding the indications for suction. However, the BTS guidelines did not deal with this issue.

As regards referral of patients to a respiratory physician or thoracic surgeon, $69 \%$ would refer in event of a PAL with a re-expanded lung and $79 \%$ would refer in event of a PAL with failure of re-expansion of the lung. In the case of continued bubbling or failure of expansion of the lung early referral is suggested in the guidelines. For non-respiratory physicians, a median of 5.0 days of intercostal drain failure would be tolerated before referral for further intervention (mean 5.2 days, range 0-24 days) while respiratory physicians had a longer tolerance, 
Table 4 Timing for removal of intercostal chest drain (ICD)

\begin{tabular}{lccr}
\hline Time to remove ICD & $\begin{array}{l}\text { Respiratory } \\
\text { physicians (\%) }\end{array}$ & $\begin{array}{l}\text { Non-respiratory } \\
\text { physicians (\%) }\end{array}$ & $\begin{array}{l}\text { All physicians } \\
\text { (\%) }\end{array}$ \\
\hline As soon as ICD stops bubbling, remove & 5.2 & 6.2 & 5.9 \\
As soon as ICD stops bubbling, clamp for 1-24 hours, then remove & 21.1 & 32.3 & 29.8 \\
24 hours after ICD stops bubbling, remove & 52.6 & 29.2 & 34.5 \\
24 hours after ICD stops bubbling, clamp for 1-24 hours, then remove & 21.1 & 30.8 & 28.6 \\
Uncertain & 0 & 1.5 & 1.2 \\
\hline
\end{tabular}

A\&E consultants and no specialty specified groups are not included in this table.

with a median of 7.0 days (mean 8.1 days, range $3-14$ days). This difference is significant, $\mathrm{p}=0.001$.

\section{RECURRENT PNEUMOTHORACES}

After a second, ipsilateral pneumothorax, $42 \%$ would refer for interventional procedures to prevent recurrence. Over 90\% would refer after a third, ipsilateral pneumothorax; $48 \%$ would not refer patients who would have had a pneumothorax on each side on separate occasions and $8 \%$ would not refer patients who have had bilateral spontaneous pneumothoraces simultaneously. Thirteen per cent thought that a first pneumothorax with severe breathlessness or complete collapse of the lung would warrant referral.

\section{Discussion}

We found that many aspects of the management of spontaneous pneumothorax in Wales differed from those recommended in the guidelines. ${ }^{2}$ Surprisingly, respiratory physicians were only slightly more compliant than other physicians, a situation different from that found in tuberculosis. ${ }^{3}$

With a standard chest radiograph, estimation of the size of a pneumothorax is often inaccurate. ${ }^{45}$ There is no uniformity in the description, with resulting confusion in interpretation among observers. The BTS guidelines suggested a practical, easy, and uniform description in terms of "small, moderate, and complete pneumothoraces". But half of the respondents used percentages to describe size of pneumothorax and even among chest physicians, the recommended description was followed by only $50 \%$.

Only half of the physicians would discharge a previously fit, young adult with a small spontaneous pneumothorax who is not breathless. Only a third were prepared to discharge a patient with primary spontaneous pneumothorax which had been successfully aspirated. This is out of line with the BTS guidelines, which, provided that the patient has rapid access to medical care if required, recommend discharge in these situations, with follow up in the chest clinic. In the current conditions of bed shortages such a policy would be helpful to the hospitals, and, presumably, be preferable to the patients. Whether the reluctance to discharge was related to geography - that is, travelling time to and from the hospital-is not certain. Another reason could have been lack of reliability of the immediate postaspiration radiograph as a predictor of persistent re-expansion. ${ }^{6}$ Patients with underlying lung disease should however be observed as inpatients, even if the pneumothorax had been small, the patient asymptomatic, and initial aspiration had been successful. ${ }^{2}$

There is evidence that initial aspiration is simple, safe, and successful..$^{7-10}$ One study reports a success rate of $83 \%$ in those without underlying lung disease, and 33\% with known pre-existing lung disease. ${ }^{7} \mathrm{Re}-$ peated aspirations also result in successful resolution. ${ }^{7}$ If active intervention is required, the BTS guidelines recommend initial aspiration in patients with and without any preexisting lung disorder, but our results indicate lack of confidence in simple aspiration, especially for patients with pre-existing lung disease or with complete collapse of the lung. Only a fifth of the respondents were prepared to aspirate a completely collapsed lung in a breathless middle aged patient with chronic bronchitis and emphysema despite the finding of Harvey and Prescott that failure of this technique was not associated with the size of pneumothorax. ${ }^{9}$ But others have found higher failure rates with larger pneumothoraces, ${ }^{67}$ and it is likely that these series and the natural conservatism of physicians more strongly influence practice with large pneumothoraces.

An intercostal drain is indicated if aspiration is unsuccessful. Applying suction initially did not result in an increased success rate in a study by So and $\mathrm{Yu}^{11}$ although suction may be tried if there is a PAL. In a randomised trial of 80 trauma patients, the chest tube time was shorter with suction compared with a simple under water seal (72.2 v 92.2 hours, $\mathrm{p}=0.013) .{ }^{12}$ Otherwise there are few data about suction for an intercostal drain in spontaneous pneumothoraces: further controlled studies are required.

If intercostal drains are removed too soon, there is a chance of lung recollapse. ${ }^{11}$ However, a third of physicians would not wait for it to stop bubbling for 24 hours before removing or clamping the tube. Although clamping of the intercostal drain risks conversion to a tension pneumothorax, ${ }^{13} 14$ about $60 \%$ indicated that they would clamp the tube before removal. The BTS guidelines recommend that chest drains should be removed 24 hours after they have stopped bubbling and discourage the practice of clamping. ${ }^{2}$

The BTS also recommends that a thoracic specialist opinion be sought early should 
the lung fail to inflate or there is PAL despite an intercostal drain, ${ }^{2}$ but only $69 \%-$ $78 \%$ indicated that they would refer patients or seek a thoracic opinion in event of failure. The reason for such reluctance is not clear. Possibilities include lack of time in hospitals where there is only one thoracic physician and scarcity or remoteness of thoracic surgeons.

The duration of failure of an intercostal drain that was tolerated varied from 0 to 24 days. Respiratory physicians tolerated PAL significantly longer than non-respiratory physicians, though the range was smaller. Advice on timing for surgical intervention is not given in the guidelines. ${ }^{2}$ The conventional recommendation for PAL has been to wait for between five and 14 days with an intercostal drain before further interventional procedures. ${ }^{15}{ }^{16}$ In one retrospective study, out of 73 patients in whom PAL developed (arbitrarily defined as continued air leak at two days after tube insertion), 43 resolved with continued tube drainage. Of these 43 patients, the median time of resolution of the PAL was seven days for those with normal lungs and 11 days for those known to have underlying lung disease $(p=0.05) \cdot{ }^{17}$ If the intercostal drain has not been successful after 48 hours, some have suggested that surgical intervention at this time would result in lower recurrence rates and possibly a shorter stay in hospital. ${ }^{18}{ }^{19}$ Late interference may result in more pleural adhesions and infection and hence further difficulties with surgery, detrimentally affecting the clinical outcome. ${ }^{20}$ Video assisted thoracoscopic surgery may reduce the threshold for intervention.

In conclusion, our study indicates that management by consultant physicians and $\mathrm{A} \& \mathrm{E}$ physicians in Wales correlates poorly with the current BTS guidelines. ${ }^{2}$ There is considerable variation in initial treatment and in referral pattern. There is reluctance to discharge patients with small primary spontaneous pneumothoraces that do not warrant active treatment and patients with primary pneumothoraces who have been successfully aspirated. There is also reluctance to aspirate secondary and/or large pneumothoraces. Management of intercostal drains can be improved: the practice of clamping is still widespread, and the tubes are often removed too soon after they have stopped bubbling. Although it is possible that practice in the UK as a whole is more in keeping with the BTS guidelines, recent reports suggest otherwise: one from a tertiary cardiothoracic centre in London found that only $25 \%$ of their referrals had the pneumothorax aspirated initially, with $69 \%$ having a chest drain inserted from the outset. ${ }^{21}$ Another, from Northern Ireland, mentioned that only $11 \%$ of 65 patients had aspiration as initial treatment and that in 12 of 50 cases the intercostal drain was clamped. ${ }^{22}$ In Liverpool, only $21 \%$ of episodes were managed as recommended by the BTS guidelines. ${ }^{23}$ Contrary to their experience, we did not find better compliance by A\&E consultants compared with respiratory physi-

\section{Learning points}

- Successfully aspirated uncomplicated primary spontaneous pneumothoraces may not need admission.

- If active treatment is warranted, uncomplicated pneumothoraces should be managed initially by percutaneous aspiration.

- The practice of clamping intercostal chest drains should be discouraged.

- The British Thoracic Society guidelines should be widely propagated in each acute hospital.

cians and we would not agree with their suggestion that compliance would improve if initial management were to be left with $A \& E$ staff. $^{23}$

The 1993 BTS guidelines at present advise on which patients require admission, on drainage procedures, on further management, on referral to a respiratory specialist, and on follow up arrangements. Areas such as indications for suction, surgery, and chemical pleurodesis and the timing of these interventions are not covered. Evidence is scanty in some of these areas and further studies are required, but we feel that the guidelines could be expanded to cover these areas and should be propagated more widely, even among respiratory physicians.

1 Melton JL, Hepper NG, Offord KP. Incidence of spontaneous pneumothorax in Olmsted County, Minnesota: 19501974. Am Rev Respir Dis 1979;120:1379-82.

2 Miller AC, Harvey JE. Guidelines for the management of spontaneous pneumothorax. BMF 1993;307:114-6.

3 Ormerod LP, Bentley C. The management of pulmonary tuberculosis notified in England and Wales in 1993. F R Coll Physicians Lond 1997;31:662-5.

4 Rhea JT, DeLuca SA, Greene RE. Determining the size of pneumothorax in the upright patient. Radiology 1982;144: $733-6$

5 Engdahl O, Toft T, Boe J. Chest radiograph — a poor method of determining size of a pneumothorax. Chest 1993;38:73743.

6 Ansari S, Seaton D. Can the chest radiograph predict early outcome of spontaneous pneumothorax? Eur Respir F 1996; 9(suppl 23):211.

7 Archer GJ, Hamilton AAD, Upadhyay R, et al. Results of simple aspiration of pneumothoraces. $\mathrm{Br} \mathcal{F}$ Dis Chest 1985;79:177-82

8 Raja OG, Lalor AJ. Simple aspiration of spontaneous pneumothorax. Br 7 Dis Chest 1981;75:207-8.

9 Harvey J, Prescott RJ. Simple aspiration versus intercostal tube drainage for spontaneous pneumothorax in patients with normal lungs. BMF 1994;309:1338-9.

10 Andrivet P, Djedaini K, Teboul JL, et al. Spontaneous pneumothorax-comparison of thoracic drainage vs immediate or delayed needle aspiration. Chest 1995;108: 335-40.

11 So SY, Yu DYC. Catheter drainage of spontaneous pneumothorax: suction or no suction, early or late removal? Thorax 1982;37:46-8.

12 Davies JW, Mackersie RC, Hoyt DB, et al. Randomised study of algorithms for discontinuing tube thoracostomy drainage. $\mathcal{f} \mathrm{Am}$ Coll Surg 1994;179:553-7.

13 Hyde J, Graham T. Reducing morbidity from chest drains. BMF 1997;314:914-5.

14 Harris DR, Graham TR. Management of intercostal drains. Br F Hosp Med 1991;45:383-6.

15 Benson MK. Pleural disease. In: Weatherall DJ, Ledingham JGG, Warrell DA, eds. Oxford textbook of medicine. 3rd Ed. Oxford: Oxford Medical Publications, 1996: 2863-72.

16 Millard FJC, Pepper JR. Pleural disease. In: Brewis RAL, Corrin B, Geddes DM, et al, eds. Respiratory medicine. 2nd Corrin B, Geddes DM, et al, eds. Respiratory medi
Ed. Philadelphia: WB Saunders 1995: 1554-79.

17 Mathur R, Cullen J, Kinnear WJM, et al. Time course of Mathur R, Cullen J, Kinnear WJM, et al. Time course of
resolution of persistent air leak in spontaneous pneumothorax. Respir Med 1995;89:129-32. 
18 Parry GW, Juniper ME, Dussek JE. Surgical intervention in spontaneous pneumothorax. Respir Med 1992; 86: $1-2$.

19 Schoenenberger RA, Haefeli WE, Weiss P, et al. Timing of invasive procedures in therapy for primary and secondary spontaneous pneumothorax. Arch Surg 1991;126: 764-6.

20 Waller DA, McConnell SA, Rajesh PB. Delayed referral reduces the success of video-assisted thoracoscopic surgery for spontaneous pneumothorax. Respir Med 1998;92: $246-9$.
21 Shah SS, Cohen AS, Magee PG, et al. Surgery remains a late and under-utilised therapeutic option in the management of spontaneous pneumothorax-should the British Thoracic Society guidelines be revised? Thorax 1998;53(suppl 4): A52.

22 Cortney PA, McKane WR. Audit of the management of spontaneous pneumothorax. Ulster Med f 1998;67:41-3.

3 Soulsby T. British Thoracic Society guidelines for the management of spontaneous pneumothorax: do we comply with them and do they work? f Accid Emerg Med 1998;15: $317-21$

\section{Medical Anniversary}

Hans Krebs, 25 August 1900

(Sir) Hans Krebs (1900-81) was born in Hildesheim, near Hannover, son of an ear, nose, and throat surgeon and became MD Hamburg in 1925. He received a biochemical basis with Otto Warburg, clinical training with Tannhauser in Freiburg, and then joined Sir Frederick Gowland Hopkins in Cambridge in 1933. He received the Nobel Prize for physiology and medicine (1953), and became Professor of Biochemistry at Oxford (1954). He will be remembered for the fundamental Krebs cycle, by which most of our food is oxidised to carbon dioxide and water. He died on 23 November 1981 in Oxford.-D G Fames 